\title{
Observation of $\mathrm{S}=+1$ narrow resonances in the system $K_{s}^{0} p$ from $\mathrm{p}+\mathrm{C}_{3} H_{8}$ collision at $10 \mathrm{GeV} / \mathrm{c}$
}

\author{
P.Zh.Aslanyan ${ }^{a, b}$, V.N.Emelyanenko ${ }^{a}$, G.G. Rikhkvitzkaya ${ }^{a}$ \\ (a) Joint Institute for Nuclear Research \\ (b) Yerevan State University \\ †E-mail: petros@spp.jinr.ru
}

\begin{abstract}
The $2 \mathrm{~m}$ propane bubble chamber experimental data have been analyzed to search for an exotic baryon state, the $\Theta^{+}$-baryon, in the $p K_{s}^{0}$ decay mode for the reaction $\mathrm{p}+\mathrm{C}_{3} H_{8}$ at $10 \mathrm{GeV} / \mathrm{c}$. The $p K_{s}^{0}$ invariant mass spectrum shows resonant structures with $M_{K_{s}^{0} p}=1540 \pm 8,1613 \pm 10,1821 \pm 11 \mathrm{MeV} / c^{2}$ and $\Gamma_{K_{s}^{0} p}=9.2 \pm 1.8,16.1 \pm 4.1$, $28.0 \pm 9.4 \mathrm{MeV} / c^{2}$. The maximal statistical significance of these peaks have been estimated as $5.5 \sigma, 4.8 \sigma$ and $5.0 \sigma$, respectively.
\end{abstract}

\section{Introduction}

Recent experimental efforts were largely motivated by Diakonov, Petrov and Polyakov [1] who studied antidecuplet baryons using the chiral soliton (Skyrme) models. The lightest member of the pentaquarks antidecuplet, $\Theta^{+}(1530)$-baryon predicted in [1], has positive strangeness, the mass of $\mathrm{M}=1530 \mathrm{MeV} / \mathrm{c}^{2}, 1 / 2$ spin and even parity. Jaffe and Wilczek have suggested an underlying quark model structure of this state [2]. There are other theoretical simulations which have predicted for this state $[3,4,5]$. The rotational states of the $\mathrm{S}=+1 \Theta^{+}$baryons are shown in the paper, by David Akers[5] . Experimental evidence for $\Theta^{+}(1530)$-baryon with positive strangeness has come recently from several experimental groups (LEPS[6]], DIANA-ITEP [7], CLAS [8], SAPHIR [9], HERMES [10], SVD-2 experiment IHEP [11]. The reaction $\mathrm{CC} \rightarrow p K_{s}^{0}+\mathrm{X}$ with the propane bubble chamber technique has observed a resonant structure in the $\mathrm{p} K_{s}^{0}$ invariant mass spectrum with $\Theta^{+}(1532)$ [12].

\section{Method}

A reliable identification of the above mentioned resonance needs to use $4 \pi$-detectors and high precision measurements of the sought objects. The $2 \mathrm{~m}$ propane bubble chamber is the most suitable instrument for this purpose $[13,14]$. The experimental information of more than $2^{*} 350000$ stereo photographs are used to select the events with $V^{0}$ strange particles - The based on the program GEOFIT[15] is used to measure the kinematics parameter of tracks - $\mathrm{p}, \operatorname{tg} \alpha, \beta$ in the photographs. The relative error of measuring momentum $\mathrm{p}$ and the average length $\mathrm{L}$ of charged particles are

$<\Delta p / p>=2.1 \%,<L>=12 \mathrm{~cm}$ for stopping particles;

$<\Delta p / p>=9.8 \%,<L>=36 \mathrm{~cm}$ for not stopping particles.

The mean values of measurement errors for the dip and azimuthal angles are relatively equal to $\langle\Delta$ tg $\alpha\rangle=0.0099 \pm 0.0002$ and $\langle\Delta \beta\rangle=0.0052 \pm 0.0001$ (rad.).

The estimate of ionization, the peculiarities of the end track points of the stopping particles(protons, $K^{ \pm}$) allowed one to identify them. Protons could be identified over the following momentum range: $0.150 \leq p \leq 0.900 \mathrm{GeV} / \mathrm{c}$. In the second momentum range $p>$ 
$0.900 \mathrm{GeV} / \mathrm{c}$ protons couldn't be separated from other particles. Therefore, the experimental information has been analyzed similarly two separate ranges.

\subsection{Identification of $\Lambda$ and $K_{s}^{0}$}

The events with $V^{0}\left(\Lambda\right.$ and $\left.K_{s}^{0}\right)$ were identified using the following criteria $[16,17]$ :

1)two-prong stars from the photographs were selected according to $\Lambda \rightarrow \pi^{-}+p$, neutral $K_{s} \rightarrow \pi^{-}+\pi^{+}$or $\gamma \rightarrow e^{+}+e^{-}$hypothesis ; 2) $V^{0}$ stars should have the effective mass of $K_{s}^{0}$ and $\left.\Lambda ; 3\right)$ these $V^{0}$ events are directed to some vertices(complanarity); 4) they should have one vertex a three constraint fit for the hypothesis with $M_{K}, M_{\Lambda}$ at $\chi^{2}<12$ and a momentum limit of $K_{s}^{0}$ and $\Lambda$ greater than 0.1 and $0.2 \mathrm{GeV} / \mathrm{c}$, respectively; 5)The analysis has shown[17] that the events with undivided $\Lambda K_{s}^{0}$ were appropriated events as $\Lambda$.

Figures.1a,c and 1b,d show the effective mass distribution of $\Lambda$ (8657-events), $K^{0}(4122$ events) particles and their $\chi^{2}$ from kinematics fits, respectively, produced from the beam protons interacting with propane targets. The measured masses of these events have the following Gaussian distribution parameters $\left\langle M\left(K_{s}\right)\right\rangle=497.7 \pm 3.6$, s.d. $=23.9 \mathrm{MeV} / c^{2}$ and $\langle M(\Lambda)\rangle=1117.0 \pm 0.6$, s.d. $=10.0 \mathrm{MeV} / c^{2}$. The masses of the observed $\Lambda, K_{s}^{0}$ are consistent with their PDG values [18]. The effective mass of the $\Theta^{+} \rightarrow K^{0} p$ system, like that of the $\Lambda \rightarrow \pi^{-} p$ system, is measured to a precision of $\left\langle\Delta M_{\left(K_{s}^{0} p\right)} / M_{\left(K_{s}^{0} p\right)}>\approx 1.1 \%\right.$. Then the effective mass resolution of $K^{0} p$ system was estimated to be on the average $0.6 \%$ for identified protons with a momentum of $0.150 \leq p \leq 0.900 \mathrm{GeV} / \mathrm{c}$.

The preliminary estimate of the experimental total cross sections for $K_{s}^{0}$ production in the $\mathrm{p}^{12} \mathrm{C}$ collisions at $10 \mathrm{GeV} / \mathrm{c}$ is equal to $\sigma=3.8 \pm 0.6 \mathrm{mb}$.

\section{$3 \quad p K_{s}^{0}$ - spectrum analysis}

\section{1 $p K_{s}^{0}$ - spectrum for identified protons with a momentum of $0.350 \leq p_{p} \leq 0.900 \mathrm{GeV} / \mathbf{c}$}

The $p K_{s}^{0}$ effective mass distribution for 2300 combination is shown on Fig.2. The solid curve is the sum of the background and 4 Breit-Wigner resonance curves.

The total experimental background has been obtained by two methods. In the first method, the experimental effective mass distribution was approximated by the polynomial function after cutting out the resonance ranges because this procedure has to provide the fit with $\chi^{2}=1$ and polynomial coefficient with errors less than $10 \%$. This distribution was fitted by the eight- order polynomial. The second of the randomly mixing method of the angle between $K_{s}^{0}$ and p for experimental events is presented in [21]. Then, these background events were analyzed by using the same experimental condition and the effective mass distribution $p K_{s}^{0}$ was fitted by the eight-order polynomial. The analysis done by two methods has shown that while fitting had these distributions the same coefficient and order of polynomial.

The background for $\overline{K^{0}} p$ combinations is estimated with FRITIOF model [19, 20] and no more than $10 \%$ has been obtained. No obvious structure in $\overline{K^{0}} p$ spectrum is seen in Fig.2.

The statistical significance for the fit in Fig. 2 is calculated as NP $/ \sqrt{N B}$, where NP is the number of events corresponding to the signal on top of the fitted background and $\mathrm{NB}$ is the number of events corresponding to the background in the chosen area. There are significant enhancements in 1540,1612 and $1821 \mathrm{MeV} / c^{2}$ mass regions. There are small peaks in 1480 and $1980 \mathrm{MeV} / \mathrm{c}^{2}$ mass regions. 


\section{$3.2 p K_{s}^{0}$ - spectrum for positively charged, relativistic tracks with a momentum of $0.9 \leq p_{p} \leq 1.7 \mathrm{GeV} / \mathrm{c}$}

The $p K_{s}^{0}$ invariant mass spectrum shows resonant structure with $\mathrm{M}=1515(5.3 \sigma)$ and 1690 $\mathrm{MeV} / c^{2}(3.8 \sigma)$ in Fig.3a. No obvious structure in 1540,1610 and $1821 \mathrm{MeV} / c^{2}$ mass regions is seen in Fig3a . FRITIOF [19, 20] model shows that the average multiplicity in this range for: all positive tracks, protons and $\pi^{+}$are equal to $1.2,0.4$ and 0.8 , respectively. The background for $K_{s}^{0} \pi^{+}$and $K_{s}^{0} K^{+}$combinations are equal to $\approx 46.6 \%$ and $4.4 \%$ respectively. These observed peaks can be a reflection from resonances $\Lambda(1520)$ and $\Lambda(1700)$. The $\left(n \overline{K^{0}}\right.$ ) invariant mass spectra for events where $\pi^{+}$-meson in reactions $\mathrm{p}+\mathrm{C}_{3} H_{8} \rightarrow \pi^{+} \overline{K^{0}} n X$ was detected and its mass was substituted by the mass of neutron.

\section{3 $p K_{s}^{0}$ - spectrum for positively charged tracks with a momen- tum of $p_{p} \geq 1.7 \mathrm{GeV} / \mathrm{c}$}

The $p K_{s}^{0}$ effective mass distribution with a momentum $p_{p} \geq 1.7 \mathrm{GeV} / \mathrm{c}(3500$ combination $)$ is shown in Fig.3b. The histogram is approximated by a polynomial background curve and by 5 resonance curves taken in the Breit-Wigner form. The dashed curve is the background taken in the form of a superposition of Legendre polynomials up to the 6 -th degree, inclusive. The analysis done by two methods has shown that while fitting had these distributions the same coefficient and order of polynomial. The average multiplicity(FRITIOF) in this range for: all positive tracks, only protons and only $\pi^{+}$are equal to $1.3,0.8$ and 0.5 , respectively. Therefore the background for $K_{s}^{0} \pi^{+}$and $K_{s}^{0} K^{+}$combinations are equal to $\approx 20 \%$ and $5 \%$, respectively. The estimate of contribution for $\overline{K^{0}} p$ combinations with FRITIOF model is equal to $9 \%$. No obvious structure in $\overline{K^{0}} p$ spectrum is seen on fig. $3 \mathrm{~b}$.

There are significant enhancements in 1487, 1544, 1612 and $1805 \mathrm{MeV} / c^{2}$ mass regions. Their excess above background is 3.0, 3.9, 3.7 and 4.0 S.D., respectively. There is a small peak in $1693 \mathrm{MeV} / \mathrm{c}^{2}$ mass regions.

\subsection{The sum of $p K_{s}^{0}$ - spectrum for identified protons and posi- tively charged tracks $\left(p_{p} \geq 1.7 \mathrm{GeV} / \mathrm{c}\right)$}

The total effective mass distribution for $p K_{s}^{0}$ (5554 combination) is shown on Fig.4. The solid curve is the sum of the background and 4 Breit-Wigner resonance curves. The background was fitted by the six-order polynomial. The total experimental background(dashed histogram) with the same experimental condition is also obtained by the second method [21]. The dashed curve is the background taken in the form of a superposition of Legendre polynomials up to the 6 -th degree, inclusive.

There are significant enhancements in $1545,1616,1690,1811$ and $1980 \mathrm{MeV} / \mathrm{c}^{2}$ mass regions. Their excess above background is by the first method 6.2,6.6, 4.0, 5.0, 3.0 S.D., respectively and by the second method 5.5, 4.8, 3.6, 5.0,3.0 S.D., respectively.

\section{Conclusion}

The effective mass spectra $K_{s}^{0} p$ in collisions protons of a $10.0 \mathrm{GeV} / \mathrm{c}$ momentum with $C_{3} H_{8}$ nuclei, have led to the discovery of the peaks presented below(Table 1). Table 1 shows the width $(\Gamma)$ and the effective mass resonances which are based on the data from Fig. 2 . The 
statistical significance are the based on the data from Fig.4. The primary total cross section for $\Theta^{+}(1540)$ production in $p C_{3} H_{8}$-interactions is estimated to be $\approx 90 \mu \mathrm{b}$. 
Table 1.

\begin{tabular}{|c|c|c|c|c|}
\hline $\begin{array}{c}\text { Resonance } \\
\text { system }\end{array}$ & $\begin{array}{c}\mathrm{M} \\
\mathrm{MeV} / c^{2}\end{array}$ & $\begin{array}{c}\Gamma_{e} \\
\mathrm{MeV} / c^{2} \\
\text { Experiment }\end{array}$ & $\begin{array}{c}\Gamma \\
\mathrm{MeV} / c^{2}\end{array}$ & $\begin{array}{c}\text { significance } \\
\text { (maximal) } \\
N_{s d}\end{array}$ \\
\hline$K_{s}^{0} p$ & $1540 \pm 8$ & $18.2 \pm 2.1$ & $9.2 \pm 1.8$ & $5.5 \pm 0.5$ \\
$K_{s}^{0} p$ & $1613 \pm 10$ & $23.6 \pm 6.0$ & $16.1 \pm 4.1$ & $4.8 \pm 0.5$ \\
$K_{s}^{0} p$ & $1821 \pm 11$ & $35.9 \pm 12.0$ & $28.0 \pm 9.4$ & $5.0 \pm 0.6$ \\
\hline
\end{tabular}

\section{Acknowledgements}

The authors are very much obliged to A.N. Sisakian, A.I.Malakhov, Yu.A. Panebratsev, H.A. Vartapetian for their support of this work.

We are also greatly indebted to our colleagues A.A. Kuznetsov, E.N.Kladnitskaya,Yu.A.Troyan, M.V. Tokarev, A.H.Khudaverdyan, A.S.Danagulian, V.I. Moroz, V.V. Uzhinskii for the assistance in data processing and fruitful discussions. 


\section{References}

[1] D. Diakonov, V. Petrov, and M. Polyakov, Z. Phys. A 359 , 305 (1997).

[2] R.L. Jaffe, SLAC-PUB-1774 Talk presented at th Topical Conf. on Baryon Resonances, Oxford, Eng., Jul 5-9, 1976;arXiv:hep-ph/0307341(2003).

[3] M. Karliner and H.J. Lipkin, Phys. Lett. B, 575 , 249 (2003), arXiv:hep-ph/0307243;

[4] C. Alt et al., 3. J.Ellis, M.Karliner,M.Praszalowich, hep-ph/0401127.

[5] David Akers, arXiv.org:hep-ex/0310014.

[6] LEPS Coll., T. Nakano et al., Phys. Rev. Lett. 91 , 012002 (2003); arXiv:hep$\exp / 0301020$.

[7] DIANA Coll., V. Barmin et al., Phys. At. Nucl., 66 , 1715-1718 (2003); arXiv:hep$\exp / 0304040$.

[8] CLAS Coll., V. Kubarovsky and S. Stepanyan, presented at the Conference on the Intersections of Particle and Nuclear Physics (CIPANP2003), New York, NY, USA, May 19-24, 2003, arXiv:hep-ex/0307088.

[9] SAPHIR Coll., J. Barthet al., Phys. Lett., B 5722004.

[10] HERMES Coll., A. Airapetian et al., arXiv:hep-ex/0312044.

[11] SVD-2 experiment IHEP, A.Aleev et al.,arXiv:hep-ex/0401024 v5, (2004).

[12] A.A.Kuznetsov, R.Togoo et al., Proc. of the Mangolian Academy of Sciences,v.170, N4,p.3,2003.

[13] M.Balandin et al., Nucl.Instr. and Meth.,1963,20.p.110.

[14] A.I. Bondarenko et al., JINR Commun.,Dubna,P1-98-292,1998.

[15] N.F.Markova, et al., JINR Commun., P10-3768,1968.

[16] G.N.Agakashiev et. al., Yad. Fiz.,1986,43(2),p.366,373.

[17] E.N.Kladnitskaya, K.J.Jovchev , P1-86-166 JINR, S.G. Arakelian et al., JINR Commun., 1-82-683, 1982.

[18] Particle Data Group, K. Hagiwara et al., Phys. Rev. D. 66,010001 (2002).

[19] FRITIOF, H. Pi, Comput. Phys.Commun. 71(1992)173

[20] A.S.Galoian et al., JINR Commun., P1-2002-54, 2002.

[21] V.L.Lyuboshits at al., JINR Rapid Comm., 1995, N6(74),p209. 

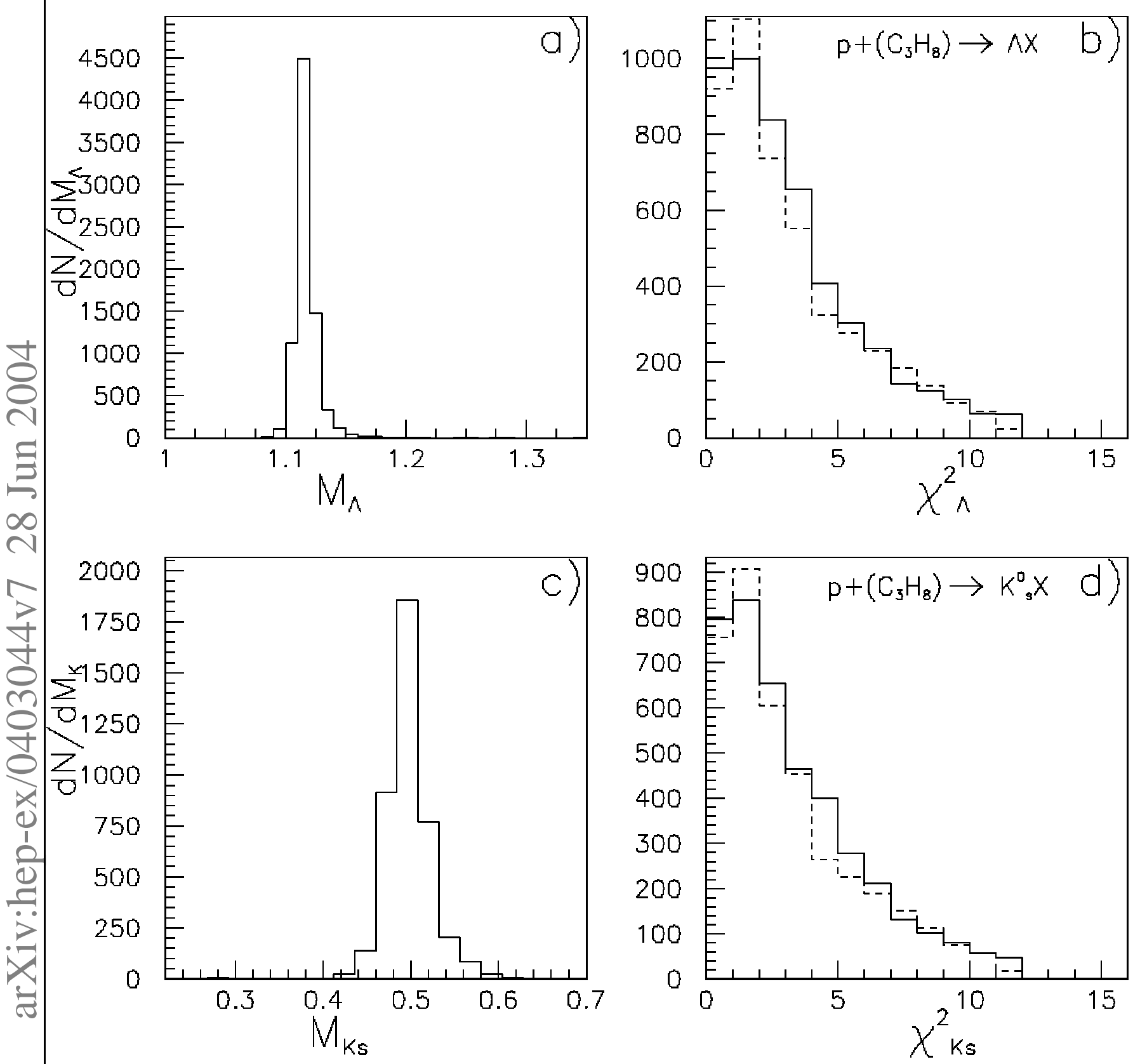

Figure 1: The distribution of experimental $V^{0}$ events produced from interactions of beam protons with propane: a) for the effective mass of $M_{\Lambda} ;$ b)for $\chi_{\Lambda}^{2}(1 V-3 C)$ of the fits via the decay mode $\left.\Lambda \rightarrow \pi^{-}+p ; \mathrm{c}\right)$ for the effective mass of $M_{K_{s}^{0}}$;d)for $\chi_{K_{s}^{0}}^{2}(1 V-3 C)$ of the fits via decay mode $K_{s}^{0} \rightarrow \pi^{-}+\pi^{+}$. The expected functional form for $\chi^{2}$ is depicted with the dotted histogram. 


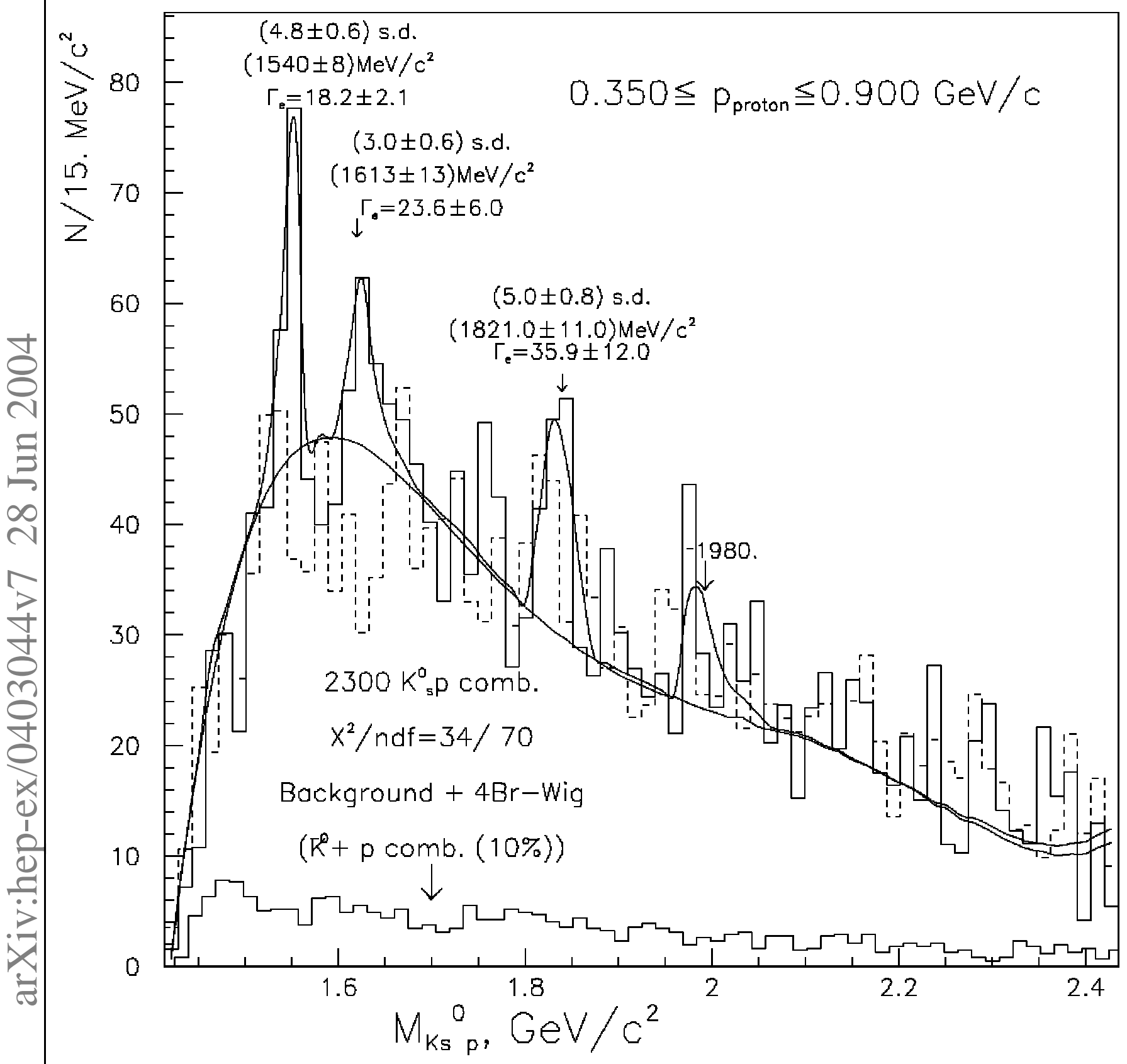

Figure 2. The $\left(p K_{s}^{0}\right)$ invariant mass spectrum with a momentum of $0.350 \leq$ $p_{p} \leq 0.900 \mathrm{GeV} / \mathrm{c}$ for identified protons in the reaction $\mathrm{p}+C_{3} H_{8} \rightarrow p K_{s}^{0}+\mathrm{X}$. The solid curve is the sum of the experimental background and 4 Breit-Wigner resonance curves. The experimental background(by the first method) taken in the form of Legendre polynomials up to the 8-th degree, inclusive. The background histogram(dashed) is obtained by the mixing method for experimental events[21]. The below histogram shows the simulated background for the spectrum of $\overline{K^{0}} p$ combinations. 


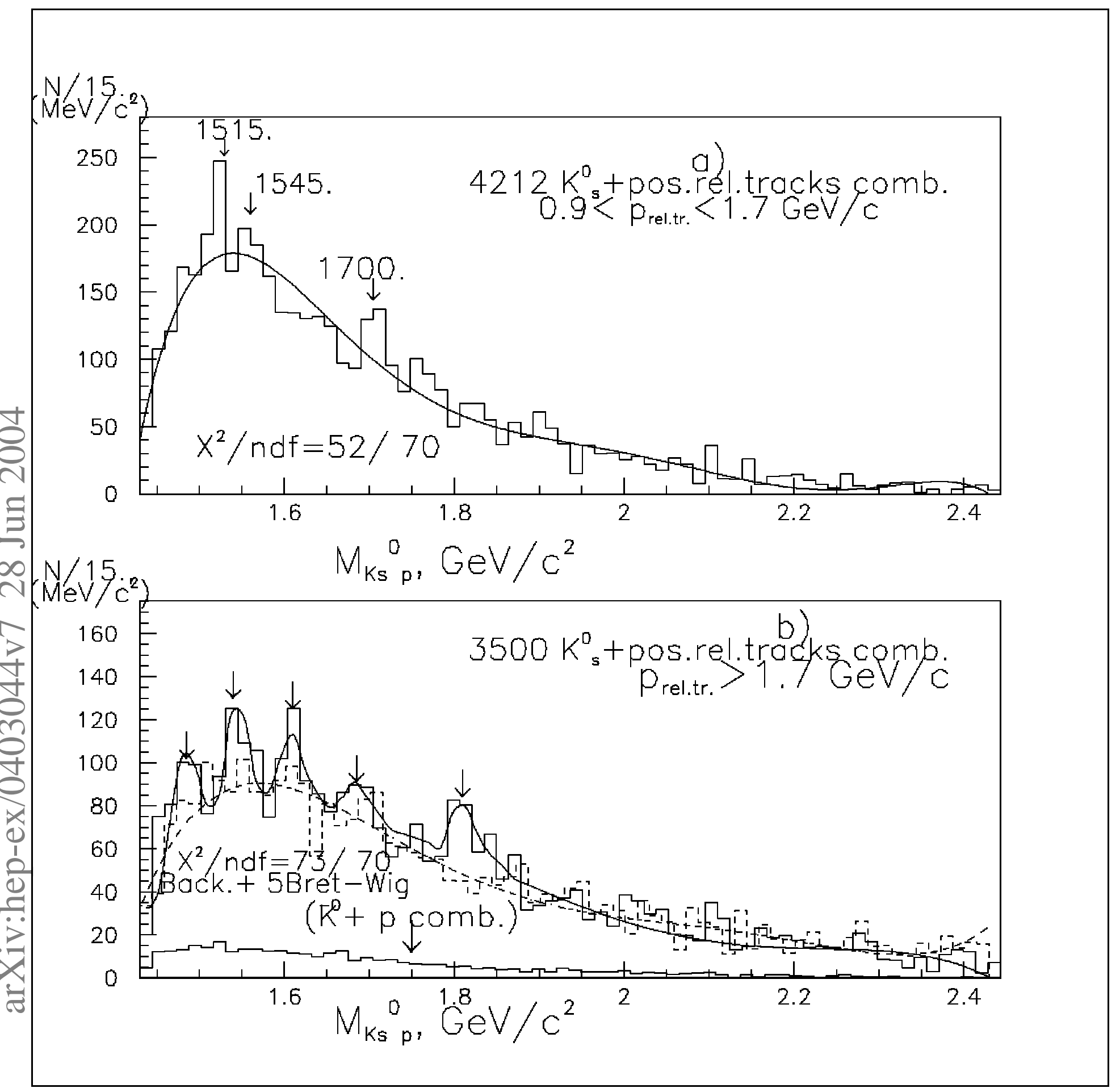

Figure 3. The $\left(p K_{s}^{0}\right)$ invariant mass spectrum in the reaction $p C_{3} H_{8} \rightarrow p K_{s}^{0}$ $+\mathrm{X}$, where protons were selected as positively charged tracks with the cuts of a momentum. a) $0.9 \leq p \leq 1.7 \mathrm{GeV} / \mathrm{c}$. The solid curve is the background distribution taken by six-order polynomial.

b) $p>1.7 \mathrm{GeV} / \mathrm{c}$. The solid curve is the sum of the experimental background(by the first method) and 5 Breit-Wigner form resonance curves. The experimental background taken in the form of Legendre polynomials up to the 6-th degree, inclusive. The dushed histogram is the experimental background[21] taken in the form of six-order polynomial(dushed curve). The below histogram shows the simulated background for the spectrum of $\overline{K^{0}} p$ combinations. 


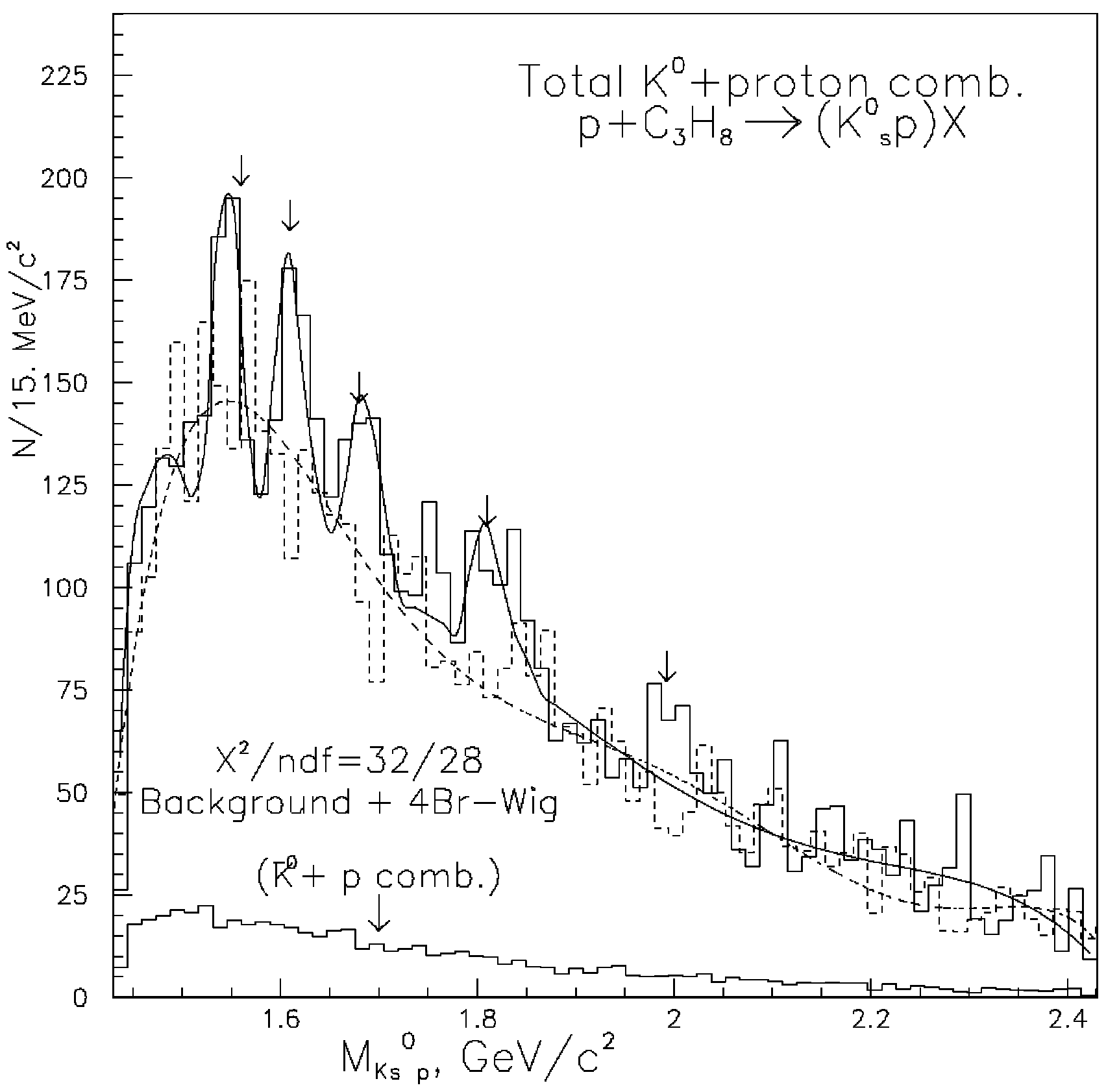

Figure 4. The sum of the effective mass distribution of $K_{s}^{0} \mathrm{p}$ - combination for protons with a momentum: $0.350 \leq p \leq 0.9 \mathrm{GeV} / \mathrm{c}^{2}$ and $p \geq 1.7 \mathrm{GeV} / \mathrm{c}^{2}$. The solid curve is the sum of the experimental background (by the first method) and 4 Breit-Wigner resonance curves. The dashed histogram is the experimental background[21] taken in the form of six-order polynomial(the dashed curve). The below histogram shows the simulated background for the spectrum of $\overline{K^{0}} p$ combinations. 